\title{
Endemic orchids of peninsular India: a review
}

\author{
Jeewan Singh Jalal ${ }^{1} \&$ J. Jayanthi ${ }^{2}$ \\ 1,2 Botanical Survey of India, Western Regional Centre, 7, Koregaon Road, Pune, Maharashtra 411001, India \\ Email: ${ }^{1}$ jeewansinghjalal@rediffmail.com (corresponding author), ${ }^{2}$ jayanthi.bsi@gmail.com
}

Orchidaceae is one of the most ecologically and morphologically diverse families of flowering plants. It is the second largest family of flowering plants in the world, comprising of about 779 genera and 22,500 species (Mabberley 2008). They have diverse habits with variously modified vegetative and floral structures. Based on their varying habits, orchids are classified as holomycotrophic or saprophytic (growing on dead and decaying matter), terrestrials (growing on ground) and epiphytic (growing on trees or shrubs). They are very sensitive to habitat degradation and fragmentation. In India, the orchid diversity is represented by 1,331 species belonging to 186 genera (Misra 2007).

The Indian subcontinent has diverse climatic regimes, forest types and habitat conditions that provides a favourable environment for accommodating diverse life forms and species. Being separated by high mountain ranges of the Himalaya in the north and in the south by Arabian Sea, Bay of Bengal and Indian Ocean, the isolation of Indian flora to a large extent helps in the evolution of endemic taxa (Nayar 1996). Geologically the drifting of the Indian subcontinent

Date of publication (online): 26 December 2012

Date of publication (print): 26 December 2012

ISSN 0974-7907 (online) | 0974-7893 (print)

Editor: Pankaj Kumar

Manuscript details:

Ms \# 03091

Received 04 February 2012

Final received 19 October 2012

Finally accepted 28 October 2012

Citation: Jalal, J.S. \& J. Jayanthi (2012). Endemic orchids of peninsular India: a review. Journal of Threatened Taxa 4(15): 3415-3425.

Copyright: @ Jeewan Singh Jalal \& J. Jayanthi 2012. Creative Commons Attribution 3.0 Unported License. JoTT allows unrestricted use of this article in any medium for non-profit purposes, reproduction and distribution by providing adequate credit to the authors and the source of publication.

Acknowledgements: The authors are thankful to Dr. Paramjith Singh, Director, Botanical Survey of India for providing facilities and support. The authors are also thankful to Dr. D.K. Singh, Additional Director, Botanical Survey of India for encouragement.

\section{OPEN ACCESS | FREE DOWNLOAD}

Abstract: The present analysis of endemic orchids shows a total account of 130 species belonging to 38 genera in peninsular India. Of these, 43 are terrestrial, 85 epiphytic and two holomycotrophic (saprophytic). The Western Ghats comprises of 123 endemic orchid species, Deccan Plateau has 29 endemic orchid species and Eastern Ghats has 22 endemic orchid species. However, in the present analysis the number of endemic species is reduced from the earlier reports because of the rapid development in the taxonomic explorations in the neighboring countries. As a result, many species were found to show extended distribution.

Key words: Deccan Plateau, endemic, Eastern Ghats, orchids, peninsular India, Western Ghats.

from the Gondwanaland through various latitudes lead to immigration and extinction of species which are engraved in the present day floristic composition (Axelrod 1971). The endemism in the flora of a country or geographical region provides an important insight into the biogeography of that region and also to the centers of diversity and adaptive evolution of the floristic components of that region (Nayar 1996). In India, the peninsular region has a high degree of endemism making it the second richest endemic centre after the Himalaya. Nayar (1977) surmised, the history of flora of peninsular India is one of the floristic impoverishments due to flow of Deccan lavas during cretaceous-eocene time and spreading aridity in Miocene-quaternary period, causing depletion of its characteristic flora leaving few relict taxa. The peninsular region is a part of Indian plate of Gondwanaland and most of the endemic plants of this region are palaeoendemics. A large concentration of endemic species is found in the tropical moist deciduous and tropical semievergreen patches of Western Ghats and to a much lesser degree in Eastern Ghats (Nayar 1996).

\section{Materials and Methods}

Peninsular India comprises of seven states viz., Andhra Pradesh, Goa, Karnataka, Kerala, Maharashtra, Odisha and Tamil Nadu and one union territory namely Pondicherry. It is bound by Vindhyan Mountains in 
the north, Arabian Sea in the west, Indian Ocean in the south and Bay of Bengal in the east. The geography of the region can be divided into three zones namely the Deccan Plateau, Eastern Ghats and the Western Ghats (Image 1). The Deccan Plateau is the largest plateau in India, making up the majority of the southern part of the country. Eastern Ghats forms a broken chain of hill ranges extending through the states of Odisha, Andhra Pradesh and Tamil Nadu. It runs north-east to southwest direction in peninsular India. Western Ghats starts near the border of Gujarat and Maharashtra, south of the Tapti River and runs approximately $1600 \mathrm{~km}$ through the states of Maharashtra, Goa, Karnataka, Tamil Nadu and Kerala ending at Kanyakumari. It is also one of the 34 Biodiversity Hotspots of the world (Myers et al. 2000). The vegetation type of peninsular India varies from tropical evergreen forest, tropical semievergreen forests, sholas, moist deciduous forests, dry deciduous forests, scrub jungles and dry savannah forests.

For the present analysis information on the endemic orchids of peninsular region was collected from literature such as Hooker (1888-1890), Blatter (1928), Fischer (1928), Cooke (1958), Santapau \& Kapadia (1966), Saldanha \& Nicolson (1976), Pradhan (1976), Bose \& Bhattacharjee (1980), Yoganarasimhan et al. (1981), Nayar et al. (1984), Rathakrishnan \& Chitra (1984), Rao (1986, 1998), Joseph (1987), Ahmedullah \& Nayar (1987), Chandrabose \& Nair (1988), Manilal (1988), Henry et al. (1989), Ansari \& Balakrishnan (1990), Keshavamurthy \& Yoganarasimhan (1990), Kumar \& Manilal (1994), Lakshminarasimhan (1996), Nayar (1996), Pullaiah (1997), Karthikeyan (2000), Gopalan \& Henry (2000), Mishra \& Singh (2001), Singh et al. (2001), Kumar et al. (2001), Yadav \& Sardesai (2002), Rao \& Kumari (2003), Manilal \& Kumar (2004), Sardesai \& Yadav (2004), Joshi \& Janarthanam (2004), Gaikwad \& Yadav (2004), Misra (2007), Misra et al. (2008), Nayar et al. (2008), Bachulkar (2010) and Narayanan et al. (2010). The online databases, namely, Govaerts et al. (2012) http://apps. Kew.org/wcsp, Tropicos (2012)

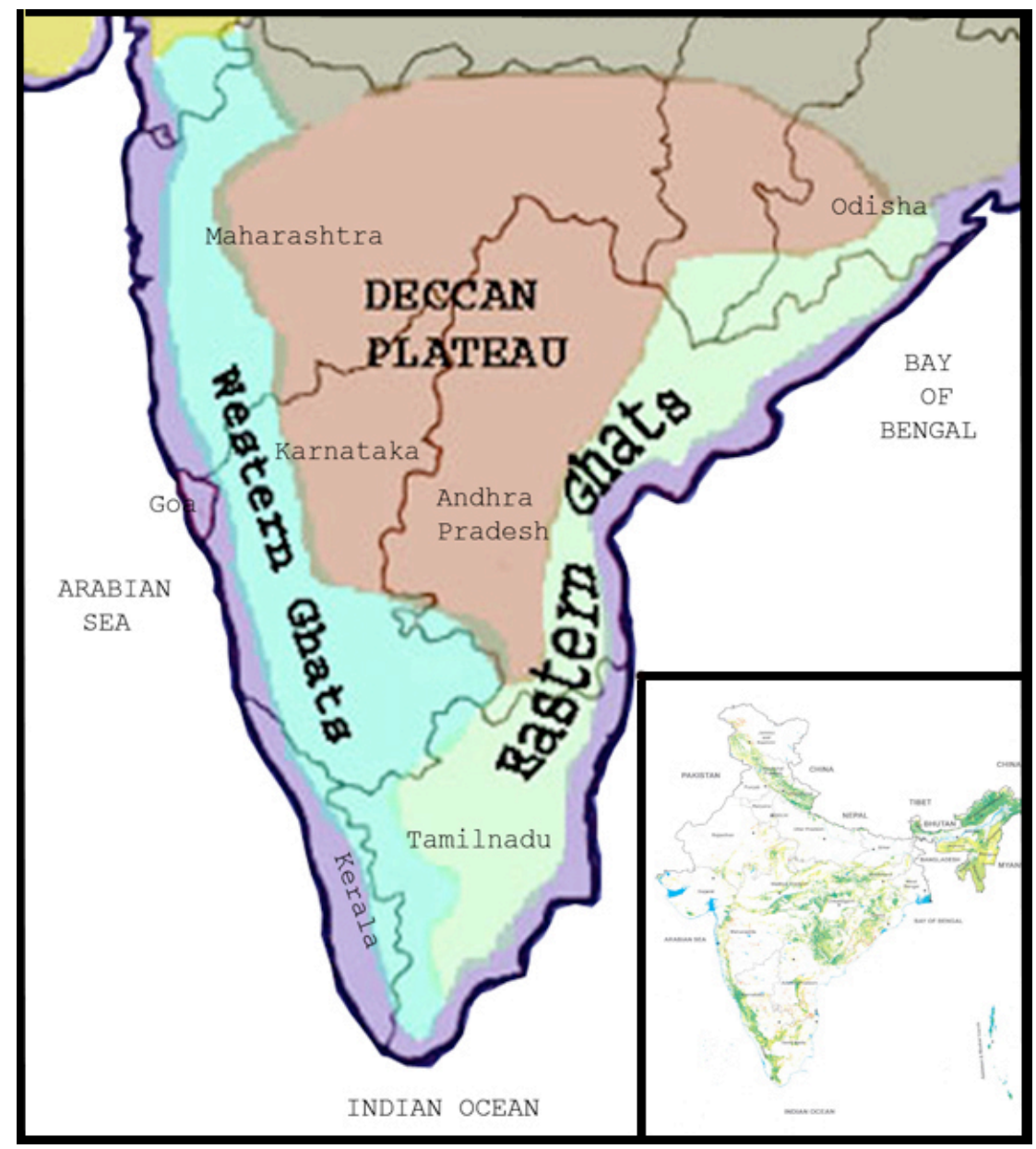

Image 1. Map of peninsular India 
www.tropicos.org, IPNI (2012) www.ipni.org, eFloras (2012) www.efloras.org were also consulted for recent updates on the plant names and distribution. Species earlier recorded as endemic but now reported from the other parts of the world, were excluded from the current list and their nomenclatural changes were also updated. The endemic orchid species are listed based on phytogeographical regions and state-wise distribution is also provided. The present work is our modest attempt to give an up-to date account of the endemic orchids of the peninsular region and to include nomenclature changes, new distributional records and new species records.

\section{Results}

Ahmedullah \& Nayar (1987) brought out the first authentic work on the endemic plants of peninsular India and estimated 123 species and 33 genera of endemic orchids from this region. While Nayar (1996) estimated 136 species, later on Kumar \& Manilal (1994) recorded 142 species belonging to 38 genera. Further, Rao (1998) estimated 126 endemic species. Singh et al. (2001) recorded 135 species and Misra (2007) recorded 160 species. So far the total endemic orchids in India are 404 (2.3\%) (Misra 2007) out of 17,500 total flowering plants, peninsular India represents $39.6 \%$ of endemic orchids out of 1,331 total number of orchids.

The present analysis resulted with a total of 130 species belonging to 38 genera endemic to peninsular India (Table 1). Of these, 43 are terrestrial, 85 are epiphytic and two are holomycotrophic. The analysis shows that the genus Habenaria (25 spp.), Oberonia

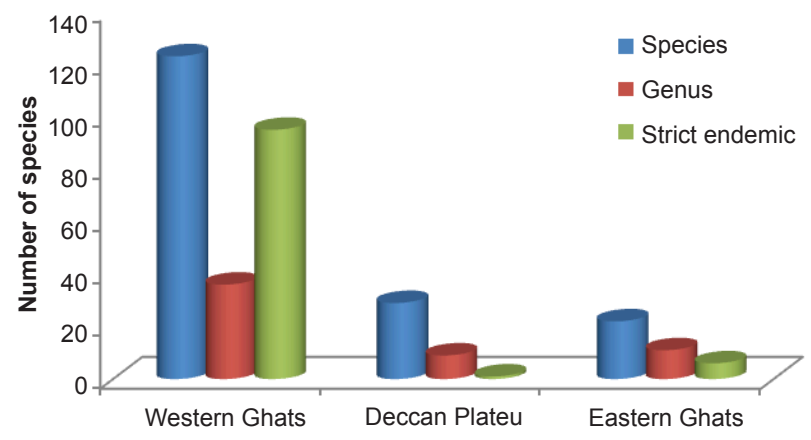

Figure 1. Species richness of endemic orchids in different regions of peninsular India

(17 spp.), Bulbophyllum (15 spp.), Dendrobium (11 spp.) and Eria (6 spp.), are among the species rich genera representing nearly $60 \%$ of total endemic orchids of peninsular India. The Western Ghats region has maximum 123 endemic orchid species followed by Deccan Plateau and then Eastern Ghats (Fig. 1). Of the total endemic orchid species of the peninsular region, $95(73 \%)$ are strict endemics to Western Ghats and five species (4\%) are restricted to Eastern Ghats. However, there are no strict endemic species in the Deccan Plateau (Fig. 1). A state wise analysis of distribution of endemic orchids shows that Kerala has a maximum number of endemic species followed by Tamil Nadu, Karnataka and Maharashtra. The states of Gujarat, Andhra Pradesh and Odisha show very poor representation of the endemic species (Fig. 2). A total of 27 orchid species earlier considered as endemic to the peninsular region are excluded from the list owing to their extended distribution in the neighbouring countries (Table 2).

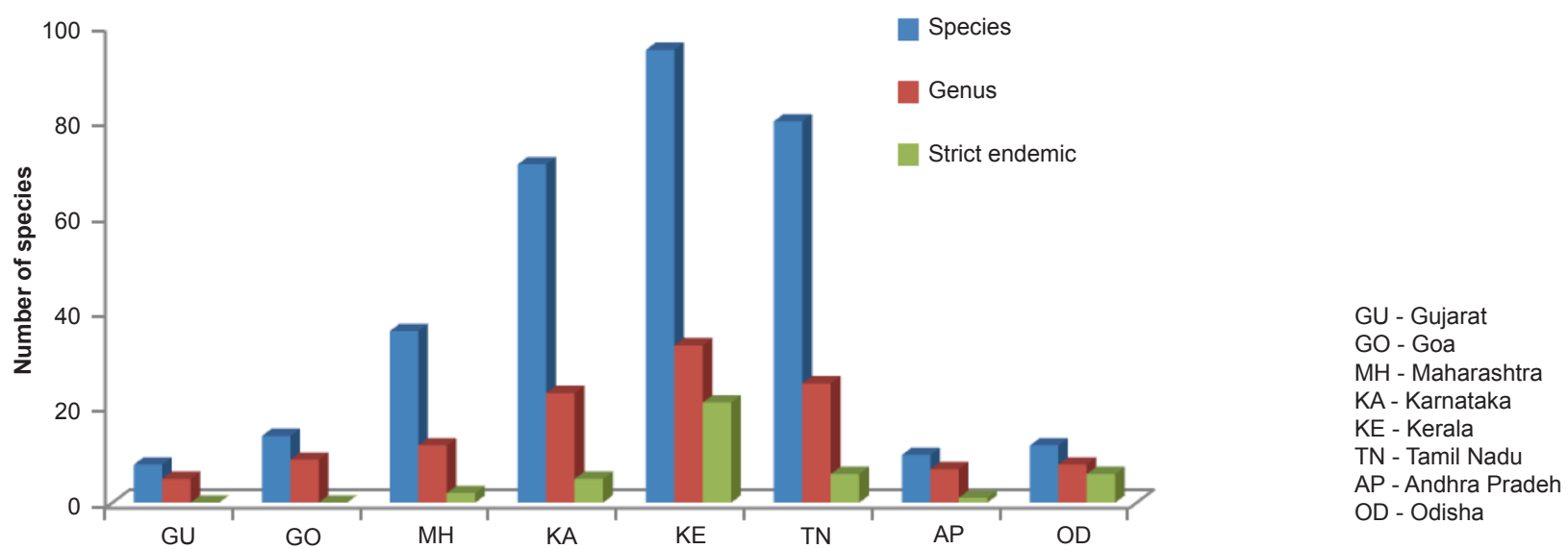

Figure 3. Species richness of endemic orchids across different states of peninsular India 
Table 1. List of endemic orchids of peninsular India

\begin{tabular}{|c|c|c|c|c|c|c|c|c|c|c|c|c|c|}
\hline \multirow{2}{*}{ Sno } & \multirow[t]{2}{*}{ Species } & \multirow[t]{2}{*}{ Habit } & \multicolumn{3}{|c|}{$\begin{array}{l}\text { Phytogeographical } \\
\text { regions of peninsular India }\end{array}$} & \multicolumn{8}{|c|}{ State wise distribution } \\
\hline & & & WG & Deccan & EG & GU & GO & MH & KA & KE & TN & AP & OD \\
\hline 1 & $\begin{array}{l}\text { Aenhenrya rotundifolia (Blatt.) } \\
\text { C.S. Kumar \& F.N. Rasm. }\end{array}$ & $\mathrm{T}$ & + & & & & & & & + & + & & \\
\hline 2 & Aerides crispa Lindl. & $E$ & + & + & & + & + & + & + & + & + & & \\
\hline 3 & Aerides maculosa Lindl. & $E$ & + & + & + & + & + & + & + & + & + & + & + \\
\hline 4 & $\begin{array}{l}\text { Brachycorythis iantha (Wight) } \\
\text { Summerh. }\end{array}$ & $\mathrm{T}$ & + & + & & & & & & + & + & & \\
\hline 5 & $\begin{array}{l}\text { Brachycorythis splendida } \\
\text { Summerh. }\end{array}$ & $\mathrm{T}$ & + & & & & & & & + & + & & \\
\hline 6 & Brachycorythis wightii Summerh. & $\mathrm{T}$ & + & & & & & & & + & & & \\
\hline 7 & $\begin{array}{l}\text { Bulbophyllum acutiflorum A. Rich. } \\
\text { = Bulbophyllum albidum (Wight) } \\
\text { Hook. f. }\end{array}$ & $E$ & + & & & & & & + & + & + & & \\
\hline 8 & $\begin{array}{l}\text { Bulbophyllum aureum (Hook. f.) } \\
\text { J.J. Sm. }\end{array}$ & $E$ & + & & & & & & & + & + & & \\
\hline 9 & $\begin{array}{l}\text { Bulbophyllum elegantulum } \\
\text { (Rolfe) J.J. Sm. }\end{array}$ & $E$ & + & & & & & & + & + & + & & \\
\hline 10 & $\begin{array}{l}\text { Bulbophyllum fimbriatum (Lindl.) } \\
\text { Rchb.f. }\end{array}$ & $E$ & + & & & & + & + & + & + & + & & \\
\hline 11 & $\begin{array}{l}\text { Bulbophyllum fuscopurpureum } \\
\text { Wight }\end{array}$ & $E$ & + & + & & & & & + & + & + & & \\
\hline 12 & $\begin{array}{l}\text { Bulbophyllum kaitiense Rchb. } \mathrm{f} . \\
\text { = Cirrhopetalum nilgherrense } \\
\text { Wight }\end{array}$ & $E$ & + & + & + & & & & + & + & + & + & \\
\hline 13 & $\begin{array}{l}\text { Bulbophyllum keralense M. } \\
\text { Kumar \& Sequiera }\end{array}$ & $E$ & + & & & & & & & + & & & \\
\hline 14 & $\begin{array}{l}\text { Bulbophyllum mysorense (Rolfe) } \\
\text { J.J. Sm. }\end{array}$ & $\mathrm{E}$ & + & + & & & & & + & + & & & \\
\hline 15 & $\begin{array}{l}\text { Bulbophyllum nodosum (Rolfe) } \\
\text { J.J. Sm. } \\
=\text { Rhytionanthos nodosum (Rolfe) } \\
\text { Garay }\end{array}$ & $E$ & + & & & & & & & & + & & \\
\hline 16 & Bulbophyllum orezii C.S. Kumar & $E$ & + & & & & & & & + & & & \\
\hline 17 & $\begin{array}{l}\text { Bulbophyllum proudlockii (King \& } \\
\text { Pantl.) J.J. Sm. }\end{array}$ & $E$ & + & + & & & & & + & & + & & \\
\hline 18 & $\begin{array}{l}\text { Bulbophyllum rheedei Manilal } \\
\text { \& C.S. Kumar = Rhytionanthos } \\
\text { rheedei (Manilal \& C.S. Kumar) } \\
\text { Garay }\end{array}$ & $\mathrm{E}$ & + & & & & & & & + & & & \\
\hline 19 & $\begin{array}{l}\text { Bulbophyllum rosemarianum } \\
\text { C.S.Kumar, P.C.S.Kumar \& } \\
\text { Saleem }\end{array}$ & $\mathrm{E}$ & + & & & & & & & + & & & \\
\hline 20 & $\begin{array}{l}\text { Bulbophyllum silentvalliensis M.P. } \\
\text { Sharma \& S.K. Srivast. }\end{array}$ & $E$ & + & & & & & & & + & & & \\
\hline 21 & Bulbophyllum tremulum Wight & $E$ & + & & & & & & + & + & + & & \\
\hline 22 & $\begin{array}{l}\text { Cheirostylis seidenfadeniana } \\
\text { C.S. Kumar \& F.N. Rasm. }\end{array}$ & $E$ & + & & & & & & & + & & & \\
\hline 23 & $\begin{array}{l}\text { Chiloschista glandulosa Blatt. \& } \\
\text { McCann }\end{array}$ & $E$ & + & & & & & & + & + & & & \\
\hline 24 & $\begin{array}{l}\text { Coelogyne mossiae Rolfe } \\
=\text { Coelogyne glandulosa var. } \\
\text { bournei S. Das \& S.K. Jain } \\
=\text { Coelogyne glandulosa var. } \\
\text { sathyanarayanae S. Das \& S.K. } \\
\text { Jain }\end{array}$ & $E$ & + & & & & & & & + & + & & \\
\hline 25 & $\begin{array}{l}\text { Coelogyne nervosa A. Rich. } \\
=\text { Coelogyne glandulosa Lindl. }\end{array}$ & $E$ & + & & & & & & + & + & + & & \\
\hline
\end{tabular}




\begin{tabular}{|c|c|c|c|c|c|c|c|c|c|c|c|c|c|}
\hline \multirow{2}{*}{ Sno } & \multirow{2}{*}{ Species } & \multirow{2}{*}{ Habit } & \multicolumn{3}{|c|}{$\begin{array}{l}\text { Phytogeographical } \\
\text { regions of peninsular India }\end{array}$} & \multicolumn{8}{|c|}{ State wise distribution } \\
\hline & & & WG & Deccan & EG & GU & GO & MH & KA & KE & TN & AP & OD \\
\hline 26 & $\begin{array}{l}\text { Conchidium filiforme (Wight) } \\
\text { Rauschert } \\
=\text { Eria dalzellii (Hook. ex Dalzell) } \\
\text { Lindl. }\end{array}$ & $E$ & + & & & & & + & + & + & + & & \\
\hline 27 & $\begin{array}{l}\text { Conchidium microchilos (Dalzell) } \\
\text { Rauschert } \\
=\text { Eria microchilos (Dalzell) Lindl. } \\
=\text { Eria tiagii Manilal, C.S. Kumar } \\
\text { \& J.J. Wood }\end{array}$ & $E$ & + & & & & + & + & + & + & + & & \\
\hline 28 & $\begin{array}{l}\text { Conchidium nanum (A. Rich.) } \\
\text { Brieger } \\
=\text { Eria nana A. Rich. } \\
=\text { Eria muscicola var. brevilinguis } \\
\text { J. Joseph \& V. Chandras. }\end{array}$ & $E$ & + & & & & & & + & + & + & & \\
\hline 29 & $\begin{array}{l}\text { Dendrobium anamalayanum } \\
\text { Chandrab., V. Chandras \& N.C. } \\
\text { Nair }\end{array}$ & $\mathrm{E}$ & + & & & & & & & + & + & & \\
\hline 30 & Dendrobium aqueum Lindl. & $\mathrm{E}$ & + & + & + & & & + & + & + & + & + & \\
\hline 31 & Dendrobium barbatulum Lindl. & $E$ & + & & & + & + & + & + & + & + & & \\
\hline 32 & $\begin{array}{l}\text { Dendrobium diodon subsp. } \\
\text { kodayarensis Gopalan \& A.N. } \\
\text { Henry }\end{array}$ & $\mathrm{E}$ & + & & & & & & & & + & & \\
\hline 33 & Dendrobium heyneanum Lindl. & $\mathrm{E}$ & + & + & & & & & + & + & + & & \\
\hline 34 & Dendrobium nanum Hook. f. & $E$ & + & + & & & & + & + & + & + & & \\
\hline 35 & Dendrobium lawianum Lindl. & $\mathrm{E}$ & + & + & & & & + & + & + & & & \\
\hline 36 & Dendrobium microbulbon A. Rich. & $E$ & + & & & + & + & + & + & + & + & & \\
\hline 37 & $\begin{array}{l}\text { Dendrobium nodosum Dalzell } \\
=\text { Flickingeria nodosa (Dalzell) } \\
\text { Seidenf. }\end{array}$ & $E$ & + & + & & & & + & + & + & + & & \\
\hline 38 & Dendrobium ovatum (L.) Kraenzl. & $E$ & + & + & + & + & + & + & + & + & + & + & \\
\hline 39 & $\begin{array}{l}\text { Dendrobium wightii A.D. Hawkes } \\
\text { \& A.H. Heller }\end{array}$ & $\mathrm{E}$ & + & & & & & & + & + & + & & \\
\hline 40 & $\begin{array}{l}\text { Didymoplexis seidenfadenii } \\
\text { C.S. Kumar \& Ormerod }\end{array}$ & $\mathrm{H}$ & + & & & & & & & + & & & \\
\hline 41 & Diplocentrum congestum Wight & $\mathrm{E}$ & + & + & & & & & + & + & & & \\
\hline 42 & $\begin{array}{l}\text { Disperis monophylla Blatt. ex } \\
\text { C.E.C. Fisch. }\end{array}$ & $\mathrm{T}$ & + & & & & & & & & + & & \\
\hline 43 & Eria albiflora Rolfe & $\mathrm{E}$ & + & & & & & & + & + & + & & \\
\hline 44 & $\begin{array}{l}\text { Eria exilis Hook. f. } \\
=\text { Porpax chandrasekharanii } \\
\text { Bhargavan \& C.N. Mohanan }\end{array}$ & $\mathrm{E}$ & + & & & & + & + & + & + & + & & \\
\hline 45 & $\begin{array}{l}\text { Eria meghasaniensis (S. Misra) } \\
\text { S. Misra }\end{array}$ & $\mathrm{E}$ & & & + & & & & & & & & + \\
\hline 46 & Eria mysorensis Lindl. & $E$ & + & & & & & + & + & + & + & & \\
\hline 47 & Eria pauciflora Wight & $E$ & + & & & & & & & + & + & & \\
\hline 48 & Eria pseudoclavicaulis Blatt. & $E$ & + & & & & & & & + & + & & \\
\hline 49 & Eulophia emilianae Saldanha & $\mathrm{T}$ & + & & & & & & + & + & & & \\
\hline 50 & Eulophia ochreata Lindl. & $\mathrm{T}$ & & + & + & + & & + & + & & & + & + \\
\hline 51 & Eulophia pratensis Lindl. & $\mathrm{T}$ & + & & & & & & & + & + & & \\
\hline 52 & $\begin{array}{l}\text { Gastrochilus flabelliformis (Blatt. } \\
\text { \& McCann) C.J. Saldanha }\end{array}$ & $E$ & + & & & & + & & + & + & & & \\
\hline 53 & $\begin{array}{l}\text { Gastrodia silentvalleyana C.S. } \\
\text { Kumar, P.C.S. Kumar, Sibi \& S. } \\
\text { Anil Kumar }\end{array}$ & $\mathrm{H}$ & + & & & & & & & + & & & \\
\hline 54 & $\begin{array}{l}\text { Habenaria barnesii Summerh. ex } \\
\text { C.E.C. Fisch. }\end{array}$ & $\mathrm{T}$ & + & & & & & & & + & + & & \\
\hline 55 & Habenaria caranjensis Dalzell & $\mathrm{T}$ & + & & & & & + & & & & & \\
\hline
\end{tabular}




\begin{tabular}{|c|c|c|c|c|c|c|c|c|c|c|c|c|c|}
\hline \multirow{2}{*}{ Sno } & \multirow{2}{*}{ Species } & \multirow{2}{*}{ Habit } & \multicolumn{3}{|c|}{$\begin{array}{l}\text { Phytogeographical } \\
\text { regions of peninsular India }\end{array}$} & \multicolumn{8}{|c|}{ State wise distribution } \\
\hline & & & WG & Deccan & EG & GU & GO & MH & KA & KE & TN & AP & OD \\
\hline 56 & Habenaria cephalotes Lindl. & $\mathrm{T}$ & + & & & & & + & + & + & + & & \\
\hline 57 & Habenaria crassifolia A. Rich. & $\mathrm{T}$ & + & + & + & & & + & + & + & + & & + \\
\hline 58 & Habenaria elliptica Wight & $\mathrm{T}$ & + & & & & & & + & + & + & & \\
\hline 59 & Habenaria elwesii Hook. f. & $\mathrm{T}$ & + & & & & & + & + & + & + & & \\
\hline 60 & $\begin{array}{l}\text { Habenaria flabelliformis } \\
\text { Summerh. ex C.E.C. Fisch. }\end{array}$ & $\mathrm{T}$ & + & & & & & & & + & & & \\
\hline 61 & $\begin{array}{l}\text { Habenaria foliosa A. Rich. } \\
=\text { Habenaria digitata var. gibsonii } \\
\text { (Hook.f.) C.E.C. Fisch. } \\
\text { = Habenaria foliosa var. foetida } \\
\text { (Blatt. \& McCann) Bennet } \\
=\text { Habenaria foliosa var. gibsonii } \\
\text { (Hook. f.) Bennet } \\
=\text { Habenaria gibsonii Hook. f. } \\
=\text { Habenaria gibsonii var. foetida } \\
\text { Blatt. \& McCann }\end{array}$ & $\mathrm{T}$ & + & + & + & & & + & + & & & & + \\
\hline 62 & $\begin{array}{l}\text { Habenaria grandifloriformis Blatt. } \\
\& \text { McCann }\end{array}$ & $\mathrm{T}$ & + & + & + & + & & + & + & + & + & & + \\
\hline 63 & Habenaria heyneana Lindl. & $\mathrm{T}$ & + & + & & & + & + & + & + & + & & \\
\hline 64 & $\begin{array}{l}\text { Habenaria hollandiana Santapau } \\
=\text { Habenaria indica C.S. Kumar } \\
\text { \& Manilal }\end{array}$ & $\mathrm{T}$ & + & + & + & & & + & + & & + & + & \\
\hline 65 & $\begin{array}{l}\text { Habenaria longicornu Lindl. } \\
=\text { Habenaria decipiens Wight }\end{array}$ & $\mathrm{T}$ & + & + & & & & & + & + & + & & \\
\hline 66 & Habenaria multicaudata Sedgw. & $\mathrm{T}$ & + & & & & + & + & + & + & + & & \\
\hline 67 & Habenaria ovalifolia Wight & $\mathrm{T}$ & + & & & & & + & + & + & + & & \\
\hline 68 & $\begin{array}{l}\text { Habenaria pallideviridis Seidenf. } \\
\text { ex K.M. Matthew }\end{array}$ & $\mathrm{T}$ & + & & & & & & & & + & & \\
\hline 69 & Habenaria panigrahiana S. Misra & $\mathrm{T}$ & & & + & & & & & & & & + \\
\hline 70 & $\begin{array}{l}\text { Habenaria panigrahiana var. } \\
\text { parviloba S. Misra }\end{array}$ & $\mathrm{T}$ & & & + & & & & & & & & + \\
\hline 71 & $\begin{array}{l}\text { Habenaria panchganiensis } \\
\text { Santapau \& Kapadia }\end{array}$ & $\mathrm{T}$ & + & & & & & + & & & & & \\
\hline 72 & $\begin{array}{l}\text { Habenaria periyarensis Sasidh., } \\
\text { K.P. Rajesh \& Augustine }\end{array}$ & $\mathrm{T}$ & + & & & & & & & + & & & \\
\hline 73 & Habenaria perrottetiana A. Rich. & $\mathrm{T}$ & + & & & & & & + & + & + & & \\
\hline 74 & Habenaria polyodon Hook. f. & $\mathrm{T}$ & + & & & & & & & & + & & \\
\hline 75 & $\begin{array}{l}\text { Habenaria ramayyana Ram. } \\
\text { Chary \& J.J. Wood }\end{array}$ & $\mathrm{T}$ & & & + & & & & & & & + & \\
\hline 76 & Habenaria rariflora A. Rich. & $\mathrm{T}$ & + & + & + & & & + & + & + & + & + & \\
\hline 77 & Habenaria richardiana Wight & $\mathrm{T}$ & + & & & & & & & + & + & & \\
\hline 78 & Habenaria suaveolens Dalzell & $\mathrm{T}$ & + & + & & & & + & + & & & & \\
\hline 79 & $\begin{array}{l}\text { Ipsea malabarica (Rchb. f.) } \\
\text { Hook. f. }\end{array}$ & $\mathrm{T}$ & + & & & & & & & + & + & & \\
\hline 80 & Liparis beddomei Ridl. & $E$ & + & & & & & & & & + & & \\
\hline 81 & Liparis biloba Wight & $E$ & + & & & & & & + & & + & & \\
\hline 82 & Liparis platyphylla Ridl. & $E$ & + & & & & & & + & & + & & \\
\hline 83 & $\begin{array}{l}\text { Liparis vestita Rchb. f. } \\
=\text { Liparis espeevijii S. Misra }\end{array}$ & $E$ & & & + & & & & & & & & + \\
\hline 84 & $\begin{array}{l}\text { Liparis walakkadensis M. Kumar } \\
\text { \& Sequiera }\end{array}$ & $E$ & + & & & & & & & + & & & \\
\hline 85 & Luisia abrahamii Vatsala & $E$ & + & & & & & & & + & & & \\
\hline 86 & $\begin{array}{l}\text { Luisia macrantha Blatt. \& } \\
\text { McCann }\end{array}$ & $E$ & + & & & & & & + & + & & & \\
\hline
\end{tabular}




\begin{tabular}{|c|c|c|c|c|c|c|c|c|c|c|c|c|c|}
\hline \multirow[t]{2}{*}{ Sno } & \multirow[t]{2}{*}{ Species } & \multirow{2}{*}{ Habit } & \multicolumn{3}{|c|}{$\begin{array}{l}\text { Phytogeographical } \\
\text { regions of peninsular India }\end{array}$} & \multicolumn{8}{|c|}{ State wise distribution } \\
\hline & & & WG & Deccan & EG & GU & GO & MH & KA & KE & TN & AP & OD \\
\hline 87 & $\begin{array}{l}\text { Malaxis crenulata (Ridl.) Kuntze } \\
=\text { Seidenfia crenulata (Ridl.) } \\
\text { Szlach. }\end{array}$ & $\mathrm{T}$ & + & & & & & & & + & & & \\
\hline 88 & $\begin{array}{l}\text { Malaxis intermedia (A. Rich.) } \\
\text { Seidenf. } \\
\text { = Seidenfia intermedia (A. Rich.) } \\
\text { Szlach. }\end{array}$ & $\mathrm{T}$ & + & & & & & & + & + & + & & \\
\hline 89 & Nervilia hispida Blatt. \& McCann. & $\mathrm{T}$ & + & & & & & & + & & & & \\
\hline 90 & $\begin{array}{l}\text { Oberonia agastyamalayana C.S. } \\
\text { Kumar }\end{array}$ & $E$ & + & & & & & & & + & & & \\
\hline 91 & Oberonia anamalayana Joseph & $E$ & + & & & & & & & & + & & \\
\hline 92 & Oberonia balakrishnanii R. Ansari & $E$ & + & & & & & & & & + & & \\
\hline 93 & Oberonia bellii Blatt. \& McCann & $E$ & + & & & & & & + & & & & \\
\hline 94 & $\begin{array}{l}\text { Oberonia brachyphylla Blatt. \& } \\
\text { McCann }\end{array}$ & $E$ & + & & & & + & & + & & & & \\
\hline 95 & Oberonia brunoniana Wight & $E$ & + & + & + & & + & + & + & + & + & + & \\
\hline 96 & $\begin{array}{l}\text { Oberonia chandrasekharanii V.J. } \\
\text { Nair, V.S. Ramach. \& R. Ansari }\end{array}$ & $E$ & + & & & & & & + & + & & & \\
\hline 97 & Oberonia josephi C.J. Saldanha & E & + & & & & & & + & & & & \\
\hline 98 & $\begin{array}{l}\text { Oberonia nayarii R. Ansari \& R. } \\
\text { Balakrishnan }\end{array}$ & $E$ & + & & & & & & + & + & + & & \\
\hline 99 & $\begin{array}{l}\text { Oberonia proudlockii King \& } \\
\text { Pantl. }\end{array}$ & $E$ & + & + & + & & & & + & & + & & + \\
\hline 100 & Oberonia platycaulon Wight & $E$ & + & & & & & + & & + & + & & \\
\hline 101 & Oberonia santapaui Kapadia & $E$ & + & + & + & & & + & + & + & + & & \\
\hline 102 & $\begin{array}{l}\text { Oberonia sebastiana B.V. Shetty } \\
\text { \& Vivek. }\end{array}$ & $E$ & + & & & & & & & + & + & & \\
\hline 103 & $\begin{array}{l}\text { Oberonia seidenfadeniana J. } \\
\text { Joseph \& Vajr. }\end{array}$ & $E$ & + & & & & & & & + & + & & \\
\hline 104 & $\begin{array}{l}\text { Oberonia swaminathanii } \\
\text { Ratheesh, Manudev \& Sujanapal }\end{array}$ & $E$ & + & & & & & & & + & & & \\
\hline 105 & Oberonia verticillata Wight & $E$ & + & + & + & & & & + & + & + & & \\
\hline 106 & $\begin{array}{l}\text { Oberonia wynadensis Sivad. \& } \\
\text { R.T. Balakrishnan }\end{array}$ & $E$ & + & & & & & & & + & & & \\
\hline 107 & Odisha cleistantha S.Misra & $\mathrm{T}$ & & & + & & & & & & & + & + \\
\hline 108 & $\begin{array}{l}\text { Paphiopedilum druryi (Bedd.) } \\
\text { Stein }\end{array}$ & $\mathrm{T}$ & + & & & & & & & + & + & & \\
\hline 109 & Peristylus brachyphyllus A. Rich & $\mathrm{T}$ & + & & & & & & + & & + & & \\
\hline 110 & Peristylus lancifolius A. Rich. & $\mathrm{T}$ & + & & & & & & + & & + & & \\
\hline 111 & $\begin{array}{l}\text { Peristylus stocksii (Hook. f.) } \\
\text { Kraenzl. }\end{array}$ & $\mathrm{T}$ & + & & & + & & + & + & & + & & \\
\hline 112 & $\begin{array}{l}\text { Pinalia polystachya (A. Rich.) } \\
\text { Kuntze }\end{array}$ & $E$ & + & & & & & & & + & + & & \\
\hline 113 & Porpax jerdoniana (Wight) Rolfe & $E$ & + & & & & + & + & + & + & + & & \\
\hline 114 & Pteroceras indicum Punekar & $E$ & + & & & & & & + & & & & \\
\hline 115 & $\begin{array}{l}\text { Pteroceras monsooniae Sasidh. } \\
\text { \& Sujanapal }\end{array}$ & $E$ & + & & & & & & & + & & & \\
\hline 116 & $\begin{array}{l}\text { Robiquetia josephiana Manilal \& } \\
\text { C.S. Kumar }\end{array}$ & $E$ & + & & & & & & & + & + & & \\
\hline 117 & $\begin{array}{l}\text { Saccolabium congestum (Lindl.) } \\
\text { Hook. f. }\end{array}$ & $E$ & + & & & & & & & + & & & \\
\hline 118 & $\begin{array}{l}\text { Schoenorchis jerdoniana (Wight) } \\
\text { Garay }\end{array}$ & $E$ & + & + & + & & & & + & + & + & + & \\
\hline 119 & $\begin{array}{l}\text { Schoenorchis latifolia (C.E.C. } \\
\text { Fisch.) Saldanha }\end{array}$ & $\mathrm{E}$ & + & & & & & & + & & & & \\
\hline
\end{tabular}




\begin{tabular}{|c|c|c|c|c|c|c|c|c|c|c|c|c|c|}
\hline \multirow[t]{2}{*}{ Sno } & \multirow[t]{2}{*}{ Species } & \multirow{2}{*}{ Habit } & \multicolumn{3}{|c|}{$\begin{array}{l}\text { Phytogeographical } \\
\text { regions of peninsular India }\end{array}$} & \multicolumn{8}{|c|}{ State wise distribution } \\
\hline & & & WG & Deccan & EG & GU & GO & MH & KA & KE & TN & AP & OD \\
\hline 120 & $\begin{array}{l}\text { Schoenorchis manilaliana M. } \\
\text { Kumar \& Sequiera }\end{array}$ & $E$ & + & & & & & & & + & & & \\
\hline 121 & $\begin{array}{l}\text { Seidenfadeniella rosea (Wight) } \\
\text { C.S. Kumar }\end{array}$ & $E$ & + & & & & & & & + & + & & \\
\hline 122 & $\begin{array}{l}\text { Smithsonia maculata (Dalzell) } \\
\text { Saldanha }\end{array}$ & $E$ & + & & & & & + & + & + & + & & \\
\hline 123 & Smithsonia straminea Saldanha & $E$ & + & & & & & & + & + & & & \\
\hline 124 & $\begin{array}{l}\text { Smithsonia viridiflora (Dalzell) } \\
\text { Saldanha }\end{array}$ & $E$ & + & & & & & + & + & + & & & \\
\hline 125 & $\begin{array}{l}\text { Taeniophyllum scaberulum } \\
\text { Hook. f. }\end{array}$ & $E$ & + & & & & & & & + & & & \\
\hline 126 & Trias bonaccordensis C.S. Kumar & $E$ & + & & & & & & & + & + & & \\
\hline 127 & Trias stocksii Benth. ex Hook. f. & $E$ & + & & & & & + & + & + & + & & \\
\hline 128 & $\begin{array}{l}\text { Xenikophyton seidenfadenianum } \\
\text { M. Kumar }\end{array}$ & $E$ & + & & & & & & & + & & & \\
\hline 129 & $\begin{array}{l}\text { Xenikophyton smeeanum } \\
\text { (Rchb.f.) Garay }\end{array}$ & $E$ & + & & & & & & + & + & + & & \\
\hline 130 & Zeuxine lindleyana A.N. Rao & $\mathrm{T}$ & & & + & & & & & & & & + \\
\hline \multicolumn{3}{|c|}{ Total } & 123 & 29 & 22 & 7 & 14 & 36 & 71 & 95 & 80 & 11 & 12 \\
\hline
\end{tabular}

T - Terrestrial; E - Epiphytic; H - Holomycotrophic; WG - Western Ghats; EG - Eastern Ghats; Gu - Gujarat; Go - Goa; MH - Maharashtra; KAKarnataka; KE - Kerala; TN - Tamil Nadu; AP-Andhra Pradesh; OD - Odisha

\section{Discussion}

Endemic taxa occur in a restricted area usually isolated by geographical or temporal barriers (Ahmedullah \& Nayar 1987). The endemic taxa occurring in such isolated/restricted areas are possible survivors of their ancient stock that occurred in continental areas which were subjected to cataclysmic geological and climatic changes (Nayar 1996). The major concentrations of endemic orchid species are found in the Western Ghats (Subramanayam \& Nayar 1974). Agasthyamalai Hills, Anamalai-High Ranges, Nilgiris-Silent Valley-Waynad-Kodagu region, Shimoga-Kanara, Mahabaleswar-Khandala and Konkan-Raigad are some of the important centers of endemism in the Western Ghats. Ninety five endemic orchid species are particularly restricted to these areas. Eastern Ghats have geological antiquity with isolated mountain ranges. The Eastern Ghats have some "ecological islands" that harbor endemic orchids. These are Ganjam-Koraput range in Odisha, Visakhapatnam Hills, Nallamalai-Cuddappah range and Tirupati Hills of Andhra Pradesh. Though Eastern Ghats possess a few rich forest patches, it has been poorly explored floristically as compared to Western Ghats. Eria meghasaniensis (S. Misra) S. Misra, Habenaria panigrahiana S. Misra, Habenaria panigrahiana var. parviloba S. Misra, Odisha cleistantha S. Misra and Zeuxine lindleyana A.N. Rao are strictly endemic to Odisha State. Aerides maculosa Lindl., Bulbophyllum kaitiense (Wight) Rchb.f., Dendrobium aqueum Lindl., Dendrobium ovatum (L.) Kraenzl., Eulophia ochreata Lindl., Habenaria crassifolia A. Rich., Habenaria foliosa A. Rich., Habenaria grandifloriformis Blatt. \& McCann, Habenaria hollandiana Santapau, Habenaria rariflora A. Rich., Oberonia brunoniana Wight, Oberonia proudlockii King \& Pantl., Oberonia santapaui Kapadia, Oberonia verticillata Wight and Schoenorchis jerdoniana (Wight) Garay have very wide distribution in the peninsular region.

The endemic orchids of the peninsular region are facing various kinds of localized threats like livestock grazing and forest fires as well as landscape-level threats such as mining, construction of roads, large as well as micro-hydal power projects, wind farms, large-scale agricultural expansion and creation of monoculture plantations. To cite an example Paphiopedilum druryi (Bedd.) Stein. which was once found in plenty in Agastyamalai Hills in southern India is now difficult to locate. 
Table. 2. Species earlier considered endemic but distributed in other regions

\begin{tabular}{|c|c|c|c|}
\hline Sno & Plant name & Distribution & References \\
\hline 1 & Anoectochilus elatus Lindl. & Sri Lanka & Fernando \& Ormerod 2008 \\
\hline 2 & $\begin{array}{l}\text { Bulbophyllum xylophyllum E.C. Parish \& Rchb. f. } \\
=\text { Bulbophyllum agastyamalayanum Gopalan \& } \\
\text { A.N. Henry }\end{array}$ & China, Myanmar, Thailand and Vietnam & Rao 1998; Govaerts et al. 2012 \\
\hline 3 & $\begin{array}{l}\text { Bulbophyllum sterile (Lam.) Suresh } \\
=\text { Bulbophyllum nilgherrense Wight }\end{array}$ & Nepal, Bangladesh, Myanmar & Lucksom 2007; Govaerts et al. 2012 \\
\hline 4 & $\begin{array}{l}\text { Chrysoglossum ornatum Blume } \\
\text { = Chrysoglossum hallbergii Blatt. }\end{array}$ & $\begin{array}{l}\text { Nepal, Cambodia, Thailand, Vietnam, } \\
\text { Sumatra, Java }\end{array}$ & $\begin{array}{l}\text { Lucksom 2007; Raskoti 2009; Govaerts } \\
\text { et al. } 2012\end{array}$ \\
\hline 5 & $\begin{array}{l}\text { Bulbophyllum fischeri Seidenf. } \\
=\text { Cirrhopetalum gamblei Hook. f. }\end{array}$ & Sri Lanka, Indo-China & $\begin{array}{l}\text { Fernando \& Ormerod 2008; Govaerts } \\
\text { et al. } 2012\end{array}$ \\
\hline 6 & $\begin{array}{l}\text { Bulbophyllum sarcophyllum (King \& Pantl.) } \\
\text { J.J. Sm. } \\
\text { = Cirrhopetalum panigrahianum (S.Misra) } \\
\text { S. Misra }\end{array}$ & Nepal and Myanmar & Lucksom 2007; Govaerts et al. 2012 \\
\hline 7 & Dendrobium herbaceum Lindl. & Bangladesh & Govaerts et al. 2012 \\
\hline 8 & Dendrobium jerdonianum Wight & Sri Lanka & Govaerts et al. 2012 \\
\hline 9 & Dendrobium panduratum Lindl. & Sri Lanka & Govaerts et al. 2012 \\
\hline 10 & $\begin{array}{l}\text { Dendrobium salaccense (Blume) Lindl. } \\
=\text { Dendrobium cathcartii Hook. f. }\end{array}$ & $\begin{array}{l}\text { Sri Lanka, Laos, Myanmar, Thailand, } \\
\text { Vietnam }\end{array}$ & $\begin{array}{l}\text { Fernando \& Ormerod 2008; Wu \& Hong } \\
\text { 2009; Govaerts et al. } 2012\end{array}$ \\
\hline 11 & Disperis neilgherrensis Wight & Sri Lanka, Thailand, Java & Kurzweil 2005; Govaerts et al. 2012 \\
\hline 12 & Eria reticosa Wight & Sri Lanka, E. Himalaya & Govaerts et al. 2012 \\
\hline 13 & $\begin{array}{l}\text { Eulophia flava (Lindl.) Hook. f. } \\
=\text { Eulophia cullenii (Wight) Blume }\end{array}$ & Nepal, Laos, Thailand, Vietnam & Wu \& Hong 2009; Govaerts et al. 2012 \\
\hline 14 & Habenaria roxburghii Nicolson & Sri Lanka & Fernando \& Ormerod 2008 \\
\hline 15 & $\begin{array}{l}\text { Habenaria digitata Lindl. } \\
=\text { Habenaria travancorica Hook. f. }\end{array}$ & $\begin{array}{l}\text { Uttarakhand, Assam, Nepal, } \\
\text { Bangladesh, Laos, Myanmar }\end{array}$ & $\begin{array}{l}\text { Khanam et al. 2001; Govaerts et al. } \\
2012\end{array}$ \\
\hline 16 & Habenaria longicorniculata Graham & Sri Lanka & Govaerts et al. 2012 \\
\hline 17 & $\begin{array}{l}\text { Hetaeria oblongifolia Blume } \\
=\text { Hetaeria ovalifolia (Wight) Hook. f. }\end{array}$ & Bangladesh, Thailand, Myanmar, Java & Govaerts et al. 2012 \\
\hline 18 & $\begin{array}{l}\text { Luisia tenuifolia Blume } \\
=\text { Luisia evangelinae Blatt. \& McCann }\end{array}$ & Sri Lanka & Fernando \& Ormerod 2008 \\
\hline 19 & $\begin{array}{l}\text { Nervilia concolor (Blume) Schltr. } \\
=\text { Nervilia scottii (Rchb.f.) Schltr. }\end{array}$ & Nepal, Bangladesh, Myanmar & Govaerts et al. 2012 \\
\hline 20 & $\begin{array}{l}\text { Oberonia wightiana Lindl. } \\
=\text { Oberonia arnottiana Wight }\end{array}$ & Sri Lanka & Fernando \& Ormerod 2008 \\
\hline 21 & $\begin{array}{l}\text { Pachystoma pubescens Blume } \\
\text { = Pachystoma hirsuta (J. Joseph \& Vajr.) C.S. } \\
\text { Kumar \& Manilal }\end{array}$ & $\begin{array}{l}\text { China, Taiwan, Nepal, Cambodia, Laos, } \\
\text { Myanmar }\end{array}$ & Govaerts et al. 2012 \\
\hline 22 & Peristylus lawii Wight & Nepal, Myanmar & Govaerts et al. 2012 \\
\hline 23 & Peristylus spiralis A.Rich. & Sri Lanka & Fernando \& Ormerod 2008 \\
\hline 24 & $\begin{array}{l}\text { Phalaenopsis mysorensis C.J. Saldanha } \\
=\text { Kingidium niveum C.S. Kumar }\end{array}$ & Sri Lanka & Fernando \& Ormerod 2008 \\
\hline 25 & $\begin{array}{l}\text { Thrixspermum musciflorum A.S. Rao \& J. Joseph } \\
=\text { Thrixspermum musciflorum var. nilagiricum J. } \\
\text { Joseph \& Vajr. }\end{array}$ & Arunanchal Pradesh & Lucksom 2007; Govaerts et al. 2012 \\
\hline 26 & Vanda wightii Rchb. f. & Sri Lanka & Fernando \& Ormerod 2008 \\
\hline 27 & $\begin{array}{l}\text { Vanilla wightii Lindl. ex Wight } \\
=\text { Vanilla wightiana Lindl. }\end{array}$ & Sri Lanka & Arenas \& Cribb 2010 \\
\hline
\end{tabular}




\section{REFERENCES}

Ahmedullah, M. \& M.P. Nayar (1987). Endemic Plants of the Indian region-Vol. 1. Peninsular India, Botanical Survey of India, Calcutta, 262pp.

Axelrod, D.I. (1971). Plate tectonics in relation to the history of angiosperm vegetation in India. Birbal Sahni Institute Paleobotany Special Publication 1: 5-18.

Ansari, R. \& N.P. Balakrishnan (1990). A revision of the India species Oberonia (Orchidaceae). Orchid Monographs 4: $1-82$.

Arenas, M.A.S. \& P. Cribb (2010). A new infrageneric classification and synopsis of the genus Vanilla Plum. ex Mill. (Orchidaceae: Vanillinae). Lankesteriana 9: 355398.

Bachulkar, M. (2010). Addition to the flora of Maharashtra. Journal of the Bombay Natural History Society 107(3): 266.

Blatter, E. (1928). A list of orchids with some new species from High Wavy Mountain (Madurai District). Journal of the Bombay Natural History Society 32: 518-523.

Bose, T.K. \& S.K. Bhattacharjee (1980). Orchids of India. Naya Prokash, Calcutta, xxiii+538pp.

Chandrabose, M. \& N.C. Nair (1988). Flora of Coimbatore. Bishen Singh Mahendra Pal Singh, Dehradun, xxxviii+398pp.

Cooke, T. (1958). Flora of Bombay. Vol. 3. (Repd. ed.) Botanical Survey of India, Calcutta, 649pp.

eFloras (2012). Published on the Internet, http://www.efloras. org by Missouri Botanical Garden, St. Louis, Missouri \& Harvard University Herbaria, Cambridge.

Fernando, S.S. \& P. Ormerod (2008). An Annotated checklist of the Orchids of Sri Lanka. Rheedea 18: 1-28.

Fischer, C.E.C. (1928). Orchidaceae In: Gamble, J.S. (ed.) Flora of Presidency of Madras. West, Newman and Adlard, London, 8: 1399-1478.

Gaikwad, S.P. \& S.R. Yadav (2004). Endemic Flowering Plant species of Maharashtra and their possible utilization, pp. 27-58. In: Pullaiah, T. (ed.). Biodiversity in India-Vol. 3. Regency Publications, New Delhi, vii +244 .

Gopalan, R. \& A.N. Henry (2000). Endemic Plants of India: CAMP for the Strict Endemics of Agasthiyamalai Hills, SW Ghats. Bishen Singh Mahendra Pal Singh, Dehradun, 476pp.

Govaerts, R., Dransfield, J., Zona, S.F, Hodel, D.R. \& A. Henderson (2012). World Checklist of Arecaceae. Facilitated by the Royal Botanic Gardens, Kew. Published on the Internet; http://apps.kew.org/wcsp/ Retrieved 201201-22.

Henry, A.N., V. Chitra \& N.P. Balakrishnan (1989). Flora of Tamilnadu. India Ser. 1: Analysis. Vol. 3, Botanical Survey of India, Coimbatore, 171pp.

Hooker, J.D. (1888-1890). Flora of British India. Vols. 5 \& 6. L. Reeve \& Co., London. 667-858 \& 1-198pp.

IPNI (2012). The International Plant Name Index. http://www. ipni.org
Joseph, J. (1987). Orchids of Nilgiris. Botanical Survey of India, Howrah, 184pp.

Joshi, V.C. \& M.K. Janarthanam (2004). The diversity of life-form type, habitat preference and phenology of the endemics in the Goa region of the Western Ghats, India. Journal of Biogeography 31: 1227-1237.

Karthikeyan, S. (2000). A statistical analysis of flowering plants of India, pp. 201-217. In: Singh, N.P., P.K. Singh, P.K. Hajra \& B.D. Sharma (eds.). Flora of India, Introductory Vol. 2, Botanical Survey of India, Calcutta, xi+469pp.

Keshavamurthy, K.R. \& S.N. Yoganarasimhan (1990). Flora of Coorg (Kodagu), Karnataka, India. Vinsat Publishers, Bangalore, vii+794pp.

Khanam, M., M.Z. Uddin, M.S. Khan \& M.A. Hassan (2001). Our present knowledge on the Terrestrial Orchidaceae. Bangladesh Journal of Plant Taxonomy 8(2): 35-49.

Kumar, C.S. \& K.S. Manilal (1994). A Catalogue of India Orchids. Bishen Singh Mahendra Pal Singh, Dehradun, iii+162pp.

Kumar, C.S., B.V. Shetty, S.S.R. Bennet, T.A. Rao, S. Molur \& S. Walker (eds.) (2001). Endemic Orchids of the Western Ghats. Conservation Assessment and Management Plan (C.A.M.P.) Workshop. Wildlife Information Liaison Development Society and Zoo Outreach Organisation, Coimbatore, India, 195pp.

Kumar, C.S., P.C. Suresh Kumar, M. Sibi \& A. Kumar (2008). A new species of Gastrodia R. Br. (Orchidaceae) from Silent Valley, Kerala, India. Rheedea 18(2): 107-110.

Kurzweil, H. (2005). Taxonomic Studies in the Genus Disperis (Orchidaceae) in Southeast Asia. Blumea 50: 143-152.

Lakshminarasimhan, P. (1996). Monocotyledons. In: Sharma, B.D., S. Karthikeyan \& N.P. Singh (eds). Flora of Maharashtra State, Series 2. Botanical Survey of India, Calcutta 794pp.

Lucksom, S.Z. (2007). The Orchids of Sikkim and North East Himalaya. S.Z. Lucksom, Siliguri, xxxx+984pp.

Mabberley, D.J. 2008. Mabberley's plant-book: a portable dictionary of plants, their classification and uses, third edition, revised, Cambridge University Press, Cambridge, xviii, $1021 \mathrm{pp}$.

Manilal, K.S. (1988). Flora of Silent Valley, Tropical Rain forests of India, Calicut, xi+398pp.

Manilal, K.S. \& C.S. Kumar (2004). Orchid Memories: A tribute to Gunnar Seidenfaden. Indian Association for Angiosperm Taxonomy (IAAT), Calicut, 265pp.

Mishra, D.K. \& N.P. Singh (2001). Endemic and Threatened Flowering Plants of Maharashtra. Botanical Survey of India, Calcutta, 414pp.

Misra, S. (2007). Orchids of India - A Glimpse. Bishen Singh Mahendra Pal Singh, Dehradun, v+402pp.

Misra, S., S.P. Panda \& D. Sahoo (2008). Orchid Flora of Andhra Pradesh, India. Bulliten of Botanical Survey of India 50(1-4): 129-146.

Myers, N., R.A. Mittermeier, C.G. Mittermeier, G.A.B. da Fonseca \& J. Kent (2000). Biodiversity hotspots for conservation priorities. Nature 403: 853-858. 
Narayanan, M.K.R., K.M. Manudev, P. Sujanapal, N.A. Kumar, M. Sivadasan \& A.H. Alfarhan (2010). Oberonia swaminathanii sp. nov. (Orchidaceae) from Kerala, India. Nordic Journal of Botany 28: 713-715.

Nayar, M.P. (1977). Changing pattern of distribution of endemic genera (Angiosperm). Journal of Economic and Taxonomic Botany 1: 99-110.

Nayar, M.P. (1996). Hot Spots of Endemic Plants of India, Nepal and Bhutan. Tropical Botanic Garden and Research Institute, Thiruvananthapuram, 252pp.

Nayar, M.P, M. Ahmed \& D.C.S. Raju (1984). Endemic and rare plants of Eastern Ghats. Indian Journal of Forestry 7 : $35-42$.

Nayar, T.S., M. Sibi, A.R. Beegam, N. Mohanan \& G. Rajkumar (2008). Flowering Plants of Kerala: Status and Statistics. Rheedea 18(2): 95-106.

Pradhan, U.C. (1976). Indian Orchids: Guide to Identification and Culture 1. Kalimpong. Premulaceae Books, 188pp.

Pullaiah, T. (1997). Flora of Andhra Pradesh-Vol. 3. Scientific Publishers, Jodhpur, IV+1349pp.

Rao, G.V.S. \& G.R. Kumari (2003). Flora of Visakapatnam District-Vol. 1. Botanical Survey of India, Kolkata, xiv+612pp.

Rao, R.S. (1986). Flora of Goa, Diu, Daman, Dadra and Nagarhaveli-Vol. 2. Botanical Survey of India, Howrah, $\mathrm{xxx}+546 \mathrm{pp}$.

Rao, T.A. (1998). Conservation of Wild Orchids of Kodagu in the Western Ghats. The Karnataka Association for the Advancement of Science, Bangalore, 242pp.

Raskoti, B.B. (2009). The Orchids of Nepal. Bhakta Bahadur Raskoti \& Rita Ale. iv+251pp.
Rathakrishnan, N.C. \& V. Chitra (1984). Distribution of endemic orchids in Karnataka, Kerala and Tamil Nadu. Journal of Economic and Taxonomic Botany 5: 10011006.

Saldanha, C.J. \& D.H. Nicolson (1976). Flora of Hassan District, Karnataka, India. Amerind Publishing Co. Pvt. Ltd., New Delhi, viii+922pp.

Santapau, H. \& Z. Kapadia (1966). The Orchids of Bombay. Manager of Publications, Delhi, vi+239pp.

Sardesai, M.M. \& S.R. Yadav (2004). Genus Habenaria Willd. (Orchidaceae) in Maharashtra, pp. 144-172. In: Pullaiah, T. (ed.). Biodiversity in India-Vol. 3. Regency Publications, New Delhi, vii+244pp.

Singh, K.P., S. Phukan \& P. Bujarbarua (2001). Orchidaceae, pp. 1735-1846. In: Singh, N.P. \& D.K. Singh (eds.). Floristic Diversity and Conservation Strategies in IndiaVol. 4. Botanical Survey of India, Kolkata. vii+2340pp.

Subramanayam, K. \& M.P. Nayar (1974). Vegetation and phytogeography of the Western Ghats, pp. 178-196. In: Mani, M.S. (ed.). Ecology and Biogeography of India. Hague, Netherland, 773pp.

Tropicos (2012). Missouri Botanical Garden, Shaw Boulevard, St. Louis, Missouri. http://www.tropicos.org.

Wu, Z. \& D. Hong (eds.) (2009). Flora of China. Missouri Botanical Garden Press, St. Louis, 570pp.

Yadav, S.R. \& M.M. Sardesai (2002). Flora of Kolhapur District. Shivaji University, Kolhapur, xiv+679pp.

Yoganarasimhan, S.N., K. Subramanyam \& B.A. Razi (1981). Flora of Chikamagalur district, Karnataka, India. International Book Distributors, Dehradun, vii $+407 \mathrm{pp}$. 